\title{
OCULAR INJURIES AND EMERGENCIES.
}

\author{
By FRANK W. LAW, M.D., F.R.C.S., \\ Asst.-Surgeon, Central London Ophthalmic Hospital; Ophthalmic Surgeon, Paddington \\ Green Children's Hospital and West Middlesex Hospital.
}

Injuries to the globe may be considered under the following headings :-

I. Damage from caustics.

2. Burns.

3. Damage by foreign bodies.

4. Contusions.

5. Wounds, which may be sub-divided into non-perforating and perforating.

If one accepts, as a definition of the term emergency, a condition of the eye which is likely to benefit from immediate treatment and of which the prognosis is made considerably worse by delay in treatment, then under the heading of emergencies one adds to the list of injuries detailed above the conditions of acute glaucoma, corneal ulceration, and embolism of the central artery of the retina. It is admittedly true that any disease or disorder demands treatment as soon as possible, and this, in the realm of ophthalmology, is more than ever true of detachment of the retina; but on account of the fact that a delay of a day or two actually makes very little difference in prognosis, and also because of the magnitude of the subject, now that operative treatment is so well (and justly) established, this condition will not receive attention in this communication.

\section{I.-Damage from Caustics.}

Damage to the globe by caustics, which are most often in a fluid state, usually comes about in the course of the patient's occupation, when this entails the handling of such bodies as strong acids or alkalis. Hot water and steam are rarely the agents; a not infrequent cause is the entry into the eye of certain depilatory agents, which have a particularly destructive effect on the cornea. There would also appear to be some specific potentiality for damage in lime, or any compound containing calcium; injury from this cause is more than usually serious, and difficult to deal with. Similarly, damage due to strong ammonia deserves the most careful and prolonged treatment.

The damage caused by caustics is of a twofold nature-immediate and remote. An active caustic burn of the cornea or conjunctiva may in itself be destructive, and cause necrosis of the tissues; as a result loss of vision ensues from the infiltration and scarring which follow the corneal burn. An equally serious source of damage is the adhesion, which may form during the healing process, between the lids and the globe, giving rise to the condition called symblepharon; this may result in disfigurement, loss of vision, and limitation of movement, or even practical immobilisation of the globe.

In the treatment of such trauma the first necessity is to remove the excess of material which may remain in the conjunctival fornices or on the globe. Immediate irrigation is carried out in the most thorough manner, care being taken fully to 
evert the upper lid, since material frequently lurks there and would escape removal if not specially looked for. Though the nature of the irrigating fluid, with one or two exceptions, is not of vast importance, it is prudent on first principals to use weak acids such as boric 2 per cent., in cases where the action of alkali is to be combated, and weak alkalis, such as sod. bicarb. 2 per cent., when an acid is the damaging element. If the nature of the corrosive fluid is not known, physiological saline solution is as good as any other. An exception to this rule is found in the case of damage by lime, whether solid or liquid; here a ro per cent. solution of neutral ammonium tartrate is the most beneficial agent that can be used. If the caustic were in solid form, a drop of cocaine should be instilled into the eye, and every particle meticulously removed with blunt forceps, after which irrigation should then be repeated.

After this preliminary treatment, fluorescine should be instilled, and the surface of the globe and lids carefully examined, to determine what area, if any, has been denuded of epithelium. This is important not only for this examination, but a similar procedure at subsequent treatments enables one to gauge the speed and extent of healing by the relative size of the staining area. If the cornea has suffered extensive damage, it is advisable to instil a mydriatic in the form of atropine solution, I per cent.; this may be in oily solution, or, better, parolein is freely instilled. Some consider that the liberal application by means of a glass rod, into the fornices, of vaseline or boracic ointment is a more effective method of preventing symblepharon-for, apart from securing healing of the damaged area, this is the most important object in treatment. In the case of a severe caustic burn prevention of symblepharon is a very difficult matter; but the difficulty in no way compares with that of the attempt to alleviate the established condition by means of a plastic operation upon the conjunctiva.

Treatment is, therefore, assiduous in order to avert the complication of symblepharon. Ankyloblepharon, or adhesion of the lids to each other, is a very rare complication. The fornices are kept thoroughly lubricated by parolein or vaseline daily; if necessary, in severe cases, a piece of material such as linen, impregnated with vaseline, may be inserted into the fornix, and the lids bandaged over it. Any burn of the skin of the lids receives, of course, the usual attention for a caustic burn of the skin. It is not unknown for subsequent scarring to cause such inversion of the lid as to give rise to the condition of trichiasis, or rubbing of the lashes on the globe-a troublesome condition which demands either destruction of the lashes by electrolysis or, if severe, a plastic operation.

\section{2.-Burns.}

The commonest cause of an actual burn of the conjunctiva or cornea by a hot body is the touching of the eye of a woman by a curling iron. This is rarely severe, the protective reflex being adequate to prevent gross damage; and since the offending instrument is usually sterile, healing is rapid. The eye should be irrigated to render the conjunctival sac clean, and the usual treatment in the form of lubrication adopted. Pain is often severe in any of these cases, and should be met by general measures.

\section{3.-Damage by Foreign Bodies.}

The presence of a foreign body under the lid or upon the globe is a very frequent source of irritation and discomfort. The variation in the amount of reaction to an embedded foreign body is remarkable; in some cases the patient 
arrives within a few minutes of its reception with a very painful, red, and watering eye ; in others a large foreign body is discovered upon the cornea in the course of the usual routine examination, the patient being unaware of its presence.

It is rarely that ordinary dust blowing into the eyes compels the patient to seek medical aid; the reflex lacrymation engendered is usually sufficient to wash the debris into the region of the inner canthus, or onwards down the lacrymal drainage paths. A piece of grit, however, or a particle of steel or emery dust, may resist this stream, and either become embedded in the cornea or bulbar conjunctiva, or come to rest beneath either lid-usually in the upper subtarsal sulcus which is a narrow groove, parallel with the lid margin, about a quarter of the way up the conjunctival surface of the lid.

Should the foreign body have struck the globe forcibly, it may become embedded at once, in which case pain is generally immediate and severe. Alternatively, it may penetrate the coats of the globe and assume an intra-ocular position, or pass through and remain in the orbital tissues; such an eventuality arises most commonly in the case of a workman using a hammer and sett on substances such as brickwork or concrete.

A patient frequently complains of "something in the eye", when this is not really the cause of the discomfort; similarly, a sore eye is complained of, and no history given, when the cause is a foreign body. An examination for this cause therefore, is always indicated in casualty work, but the discovery of a foreign body is not always easy. A search is first made in the lower fornix ; then the upper lid is everted and the conjunctival surface of lid and globe examined. The cornea is then examined, with the aid of a magnifier if necessary; a drop of fluorescine in the eye is of great assistance in this search. Finally, guided by the history, signs of penetrating damage are looked for-and these are not infrequently far less obvious than the inexperienced would suppose. Should there be a corneal, corneo-scleral, or conjunctivo-scleral wound, a hole in the iris, or a wound of the lens, the diagnosis is already made of perforating injury-whether due to retained foreign body or not remains to be decided. Should the foreign body have been of so great a size that the globe is severely disorganized, no difficulty is encountered, but it may happen that entry to the globe, through the conjunctiva and sclera especially, caused so little external damage that the most careful examination fails to arrive at the correct diagnosis.

With regard to treatment, foreign bodies under either lid should be removed by a pledglet of damp cotton-wool; an endeavour should be made to wipe a foreign body off the cornea, after cocainisation in a similar way. If this fails, a blunt instrument such as a spud should be used; lastly a sharp ophthalmic needle (such as Bowman's) may be necessary, to dig the foreign body from the cornea. If the foreign body is suspected of being very deeply embedded, its depth should be estimated by means of the slit lamp, and great caution exercised to avoid perforation of the cornea in the attempted removal, if the foreign body is deep. During the process of removal, the eye must be well illuminated, and the patient coached to co-operate by fixing a suitably placed object with the other eye, which must remain open. Failure on the patient's part to keep the eye under treatment still is almost invariably due to his forgetting to keep the other eye open.

Foreign bodies in the conjunctiva, if not merely lying on the surface, are sometimes rather difficult to remove on account of the laxity of this membrane. They may be picked off with forceps, or it may be necessary to pick up the 
foreign body and conjunctiva and snip both away with scissors; unless a large area is thus removed a suture is rarely necessary afterwards. Deeply embedded conjunctival foreign bodies which have been there a long time are best left alone in most cases; sub-conjunctival foreign bodies embedded in the sclera need careful removal, usually under theatre conditions, since a conjunctival flap should be raised, and vitreous loss may follow the withdrawal of the foreign body, should this have perforated.

The entry of a foreign body into the globe is a serious event and may result in loss of the eye and danger to the fellow eye: as such it is deserving of the utmost respect and the prognosis should be guarded. It is an advantage to localize the foreign body before attempting removal, though this cannot always be done clinically; localization by means of X-rays should be carried out-a highly technical and very nice procedure-and a careful fundus examination made in an effort to localize it visually. The removal of a non-magnetizable intra-ocular foreign body may be a matter of extreme difficulty, and does not demand discussion here; if the foreign body is magnetizable its removal is usually simpler and is accomplished by means of the magnet, the technical details of which are similarly without the scope of this paper. Suffice it to say that, in general, attempts at the removal of an intra-ocular foreign body should always be made; there are only very rare exceptions to this rule.

Perforating foreign bodies, whether retained or not, may give rise to the two obvious complications of intra-ocular hæmorrhage and intra-ocular infection; sympathetic ophthalmia is also an ever-present possibility, a few details of which will be discussed later. A less well known complication of a retained iron foreign body is the occurrence of siderosis-a condition in which the iron, dissolved by tissue fluids and elaborated into a chemical form of which the nature is so far unknown, is deposited in the form of brownish granules in various parts of the eye. Degenerative changes follow, causing destruction of vision.

\section{4.-Contusions.}

The damage caused by a contusion of the globe by a blunt instrument may be, according to the severity of the injury, anything from a simple abrasion to a ruptured globe. It is often surprising what little sign of damage is to be found in an eye after what is described as a serious accident, and what far reaching effects on vision an apparently trivial trauma may cause. It is impossible to forecast with any certainty the ultimate effect on vision of a contusion or laceration of the globe, unless this be of the very simplest variety.

In the case of a suspected corneal abrasion a drop of fluorescine should be instilled into the eye and any solution of continuity of the epithelium carefully looked for. Should this be found, the essentials are to render the surroundings as clean as possible and to prevent the lid from rubbing over the abraded surface. To this end the conjunctival sac is irrigated, and the lacrymal sac examined to eliminate the possibility of the presence of a mucocele; should this be found, one must at least wash the sac clean. Parolein, or some inert oily substance of that nature, is then instilled into the eye, and a pad and bandage applied. The danger in such a case is for the area to become infected and a corneal ulcer to arise ; should this appear to be at all likely the pupil must be dilated with atropine and the canaculi obliterated by cautery or the lachrymal sac excised. It should be noted, however, that in all cases the use of atropine in adults is attended by: potential danger in that it may cause a rise of tension in an eye predisposed 
to glaucoma; no middle-aged or elderly person with a shallow anterior chamber should have a mydriatic prescribed for home use, or at least not without warning of the danger, in such cases.

Corneal abrasions may occasionally recur in a most exasperating manner; this is the more likely to happen when the original lesion was caused by a finger nail. Measures such as scraping and carbolizing, mild X-rays, and many others have been advocated for this condition; I have found the local application of ultra-violet light to be practically infallible.

Contusion of the globe may cause an effusion of blood into the anterior chamber (hyphama); this is not in itself a serious matter, as absorption is usually rapid and complete, but the possible sequela of blood-staining of the cornea is far more serious: this is usually the result of a combination of hyphæma and raised tension, and though clearing is generally complete or nearly so, the process may take years. A similar occurrence behind the lens-hæmorrhage into the vitreousis always of more serious import, as absorption is rarely complete, and organization may occur, giving rise in time to the so-called retinitis proliferans, with its possible complication of detached retina.

The results of contusion on the iris may vary very considerably. Frequently a traumatic mydriasis follows, from which recovery may never be complete; all degrees of laceration of the pupillary margin of the iris are met with, though these are not common. A separation of the iris from the ciliary body, or iridodialysis, is more often met with; this may be accompanied by a folding of the iris forwards or backwards-anteflexion or retroflexion: rarely the iris is completely torn away from its attachment, and sinks to the bottom of the chamber. In all cases of injury to the iris except radial tears it is advisable to instil atropine, and to keep the patient at rest in bed, especially when intra-ocular hæmorrhage has occurred.

The changes in the lens which may follow contusion are concussion cataract, and dislocation. Although opacification most frequently follows a rupture of the lens capsule, by allowing access of intra-ocular fluid to the lens fibres, it may occur with an intact capsule; probably this is due to a nutritional disturbance secondary to damage of the ciliary body. In dislocation, the primary injury is that of rupture of the suspensary ligament; if this is partial the dislocation is partial also (subluxation), and is often difficult to diagnose, perhaps the only signs being a slight inequality in the depth of the anterior chamber at different places, and a tremulousness of the unsupported portion of the iris (iridodonesis). In total dislocation the lens may very rarely enter the anterior chamber; more commonly dislocation occurs posteriorly and the lens sinks to the bottom of the vitreous. The partially dislocated lens needs no treatment unless it causes a complication such as glaucoma; in this case operative relief of tension is indicated, but in that of total dislocation forwards removal of the lens becomes imperative. It is a very difficult procedure attended by considerable danger, as loss of vitreous almost always occurs.

The choroid may be ruptured by a contusion. The rupture is seen as a white streak, on account of the visibility of the underlying sclerotic, and is usually concentric with the disc margin and fairly close to it. It is only when the rupture occurs at or near the macula that disturbance of vision follows. The patient should be kept at rest in bed until absorption of the blood, extravasated as a consequence of the rupture, has occurred. 
Rupture of the retina is a rare condition. Hæmorrhages following contusion are more common; a more frequent disturbance is that referred to as commotio retina, which is probably due to œdema, and is seen ophthalmoscopally as a cloudiness of the retina, usually wide-spread. Visual recovery is as a rule complete; this is, however, not so if macular disturbance has followed the contusion. Such disturbances may manifest themselves in many forms, of which the commonest are hæmorrhage, pigmentary change, and "hole" at the macula-a condition probably consequent upon cystic degeneration following œedema. Finally, the retina may become detached after contusion; this may occur at varying intervals after the injury, and demands immediate operative interference.

A serious result of contusion by a blunt instrument is rupture of the globe. This usually occurs as a result of compression of the globe against the orbital wall; the globe gives way at its weakest part, just outside the limbus, and the rupture is concentric with this structure. Serious concomitant intra-ocular damage may occur, such as dislocation of the lens and iridodialsis, and hyphæma is common; often nothing remains but to excise the eye. In less serious cases it is possible to save the eye, though vision is usually severely affected.

Should the lens be dislocated so as to lie beneath the conjunctiva it is best left there, at least temporarily, since its attempted removal may result in considerable vitreous loss. It is seldom that suture of the sclerotic is indicated; the best treatment consists in the instillation of atropine, and rest; healing occurs often with surprising readiness, and a reasonably useful eye may result. Should there be a prolapse of uveal tissue, this should be excised and the conjunctiva sutured over the rent. Any operative interference should be conducted with the utmost care, since undue disturbance tends to the further loss of intra-ocular contents.

\section{5.-Wounds of the Eyeball.}

Wounds of the eye are satisfactorily divided into non-perforating and perforating. In any case of wound of the globe by a sharp instrument, the lids are liable to be lacerated; if this occurs, treatment by suture is usually fairly simple. Should the whole thickness of the lid be involved it is advisable to insert conjunctival sutures as well, in order to ensure that the lid is restored as nearly as possible to its former condition after healing.

Conjunctival wounds of the globe must be carefully investigated in order to make certain that no scleral perforation has occurred, for if such has happened the prognosis is, of course, gravely altered. The globe is irrigated with boric lotion or acriflavine, and the conjunctiva sutured with silk; healing usually occurs very rapidly.

Perforating wounds of the globe divide themselves into those of the cornea, sclera, and corneo-scleral junction. A perforating corneal wound is usually linear, and may be complicated by a prolapse of the iris. To determine thepresence or absence of this complication one must note the depth of the anterior chamber and the shape of the pupil-for if the iris has been swept into the wound with the outrush of aqueous, there must be some resultant distortion of the pupil. The penetration may have been deep enough to injure the lens, in which case the outlook is rendered still more serious by the occurrence of a traumatic cataract. Any prolapse of iris tissue must be excised and not replaced, and the greatest care taken to ensure that no adhesion of the iris to the wound remains. The eye is then 
atropinized and tied up, and the patient kept strictly at rest for at least a week. If the lens has been wounded the access of aqueous to this structure causes the fibres to swell, and lens matter comes forward into the anterior chamber; should this cause occlusion of the filtration angle a rise in tension may result, in which case the lens matter must be allowed exit (curette evacuation).

In the case of a large or lacerated corneal wound it is often of considerable value, after the excision of the prolapse which almost certainly will have occurred, to cover the wound with a flap of conjunctiva. This membrane is raised from the limbus, and freed from the globe by undercutting; it is then drawn across and secured by sutures so that the cornea is temporarily partly or even completely covered by the flap. The sutures generally cut out in a few days, by which time healing has occurred.

Perforating wounds of the sclera are accompanied by the loss of more or less vitreous, and if this is excessive shrinkage of the globe is likely to occur. It is rarely necessary to attempt scleral suture-a difficult and delicate procedure which may easily result in the further loss of vitreous. It is sufficient to suture the conjunctival wound and to keep the eye completely at rest in order to permit of healing under the best possible conditions. One golden rule in dealing with suspected scleral wounds is to refrain from testing the tension of the globe-for although it may help in diagnosis it will almost certainly cause more intra-ocular contents to be extruded in cases where perforation has occurred.

Wounds of the cornea-scleral junction are more difficult than others to deal with, and are more liable to set up sympathetic ophthalmia. Prolapse of the ciliary body must be excised, but it is far from easy to ensure permanent disentanglement from the wound; the proximity of the vitreous renders intra-ocular infection more likely. It is in gaping limbal wounds that the conjunctival flap proves most valuable.

The serious complications of perforating wounds of the globe are sympathetic ophthalmia and panophthalmitis. The latter is the more likely to occur if a direct path for infection to the vitreous has been opened up; evisceration of the globe is usually necessary, enucleation being avoided on account of the danger of opening up the meningeal sheaths through section of the optic nerve. Sympathetic ophthalmia is a dreaded complication of which the pathology is still quite obscure; it is impossible to discuss the matter here at all adequately. Inflammation of the other eye has been reported at intervals varying from a few weeks to many years after the injury. Iridocyclitis occurs in the injured eye, and if at all persistent should be regarded as an indication for excision; if a similar condition shows signs of developing in the second or sympathizing eye it is often a matter of extreme difficulty to decide whether excision of the exciting eye will benefit matters -for sometimes the original eye is eventually the more useful member of the two. It is safe to assume that sympathetic ophthalmia never occurs after excision, if no signs of its presence were visible before. In cases where the condition is threatened and excision is decided against, treatment consists in that of iridocyclitis in general, together with mercurial inunctions or Salvarsan injections.

Perforating injuries of the orbit may have direct or indirect consequences. Direct damage may be done to the optic nerve, or ocular muscles; or these may be involved in hæmorrhage or exudate, which may later cause interference with movement or with vision by strangulation after organization and subsequent contraction. If the causative foreign body is not sterile, orbital cellulitis may ensue, 
which demands active treatment by local heat, and early incision if suppuration results. Fractures of the skull-base may involve the orbital foramen; the optic nerve may be directly injured, or interference with vision may ensue through optic atrophy consequent upon compression by clot.

\section{6.-Non-Traumatic Emergencies.}

All the injuries mentioned in the foregoing must be regarded as ophthalmic emergencies; in addition, there are a few conditions other than injuries which must be similarly regarded.

Corneal ulceration is sometimes so sudden in onset, and causes so much pain, that immediate advice is sought by the patient. Ulcers of this kind are usually of the herpetic type, and an actual dendritic ulcer may appear so suddenly that the patient is convinced that some injury has befallen the eye. Examination with fluorescine reveals the source of the pain and discomfort; the best treatment is immediate carbolization, atropine, and a pad and bandage.

A fairly common and very serious ocular emergency is acute glaucoma. The patient is found in great pain, with an engorged eye, of which the cornea is hazy and the tension raised-usually the globe is stony hard. The pupil is half dilated, oval, and inactive or very feebly active; pain may be so severe as to cause vomiting. The first essential in treatment is to secure a fall in tension at the earliest possible moment, as delay may cause permanent damage to the optic nerve, with loss of vision; this fall is usually best achieved by the instillation of eserine, I per cent., every fifteen minutes, the application of two leeches to the temple, and hot bathings to the eye. Relief of pain and tension is often dramatically achieved by means of the diathermy current; this is applied by special electrodes for ten to fifteen minutes, at a strength of some $400-600$ milliamperes, according to the degree of heat which the patient will stand. It must not be thought that the case is settled when the acute attack has been successfully dealt with by the above measures; even if operation in the acute stage is avoided -and this is not always the case, though it is the ideal at which to aim-operation is usually necessary to render the eye safe for the future both from the insidious damage of chronic glaucoma, and from recurrence of the acute attack.

Embolism of the central artery of the retina must be regarded as an emergency, since active treatment at once may save a considerable amount of vision. The retinal anæmia causes sudden blindness, and if ophthalmoscopical examination is made at once-a rare possibility - the arteries are seen to be much reduced in size, the smaller ones being quite invisible. In a short time the retina becomes opaque through œedema, the lack of this at the thinnest part-the macula-causing the structure to appear bright red by comparison. The blockage may not be complete, in which case some movement of blood may be visible in the vessels, sometimes in a reversed direction to the normal.

Treatment is only valuable if available at once, and is directed towards encouraging movement of the clot into a less important branch of the vessel. To this end, massage of the globe should be performed; if no result ensues paracentesis of the cornea is carried out, the lowering of intra-ocular tension thus caused being sometimes instrumental in moving the clot on account of the temporary withdrawal of support from the arterial wall. Unless the case is seen, however, within a very few hours of the occurrence of embolism, any treatment is bound to be unavailing. 